\title{
VARIAÇÃO DO NÍVEL DO MAR COM BASE NA ANÁLISE ORGANOFACIOLÓGICA DE UM TESTEMUNHO DO TALUDE CONTINENTAL RECENTE DA BACIA DE CAMPOS, RJ, BRASIL
}

\author{
FERNANDO MACHADO LAPLACE, ANTONIO DONIZETI DE OLIVEIRA, JOÃO GRACIANO \\ MENDONÇA FILHO, RENATA BRENAND ALVARENGA DAS CHAGAS \\ LAFO/DEGEO/IGeo/UFRJ, Ilha do Fundão, 21949-900, Rio de Janeiro, RJ, Brasil.fmlaplace@yahoo.com.br, \\ donizeti@geologia.ufrj.br,graciano@geologia.ufrj.br,renata.brenand@yahoo.com.br
}

\section{TAÍSSA RÊGO MENEZES}

GEO/CENPES/PETROBRÁS, Ilha do Fundão, 21949-900, Rio de Janeiro, RJ, Brasil. taissa.menezes@petrobras.com.br

\author{
ELISAMARA SABADINI SANTOS \& JOALICE DE OLIVEIRA MENDONÇA \\ LAFO/DEGEO/IGeo/UFRJ, Ilha do Fundão, 21949-900, Rio de Janeiro, RJ, Brasil. \\ elisamara@geologia.ufrj.br, joalice@geologia.ufrj.br
}

\begin{abstract}
SEA LEAVEL CHANGES BASED ON ORGANIC FACIES ANALYSIS IN A SEDIMENTARY CORE FROM CONTINENTAL SLOPE OF CAMPOS BASIN, RJ, BRAZIL. Eighty-five samples collected in a core belonging to the BU91-GL-77, well located in the continental slope of Campos Basin, were analyzed by microscopic techniques (palynofacies) and organic geochemistry (TOC). The objective was to provide information about the organic composition of the sedimentary section analyzed. The variation in organic composition found in this sedimentary section can be a reflection of sea level and climate changes that not only affected the production, transport and preservation of organic matter but also its supply and sedimentation in Campos Basin's recent continental slope. A ternary diagram was used for the paleoenvironmental characterization of the succession studied. It was prepared from the abundance of data on the three main groups of organic matter used to define nine paleoenvironments based on marine palynofacies data. Using these diagrams, it was possible to draw the curve of relative sea level based on the proximal-distal trends during the sedimentation of particulate organic components. The data point to a general trend of marine transgression marked by small cycles of lowering/rising of the relative sea level caused by variations in sediment influx, reflecting the climatic fluctuations during the Quaternary.
\end{abstract}

Key words: palynofacies, sea level changes, Quaternary, Campos Basin.

\begin{abstract}
RESUMO - Um total de 85 amostras coletadas em um testemunho de sondagem correspondente ao poço BU91-GL-77 localizado no talude continental da bacia de Campos foram analisadas por técnicas microscópicas (palinofácies) e organogeoquímicas (COT) fornecendo informações sobre a composição orgânica da seção sedimentar analisada. A variação organocomposicional encontrada nesta seção sedimentar pode ser um reflexo das oscilações do nível do mar e mudanças climáticas que influencia tanto na produção, transporte e preservação quanto no suprimento e sedimentação da matéria orgânica no talude continental recente da bacia de Campos. Para a caracterização paleoambiental da sucessão estudada, utilizou-se diagrama ternário confeccionado a partir dos dados de abundância relativa dos três grupos principais de matéria orgânica que contém a definição de nove paleoambientes baseados nos dados de palinofácies marinha. Por meio destes diagramas foi possível traçar a curva do nível relativo do mar baseado nas tendências proximais-distais durante a sedimentação dos componentes orgânicos particulados. Observou-se tendência geral de transgressão marinha marcada por pequenos ciclos de rebaixamento/elevação do nível relativo do mar em virtude das variações do influxo sedimentar, reflexos estes das flutuações climáticas do Quaternário.
\end{abstract}

Palavras chave: palinofácies, variações do nível do mar, Quaternário, bacia de Campos.

\section{INTRODUÇÃO}

O intervalo de tempo representado pelo Pleistoceno $(1,81$ Ma, Gibbard \& Kolfschoten, 2004; Lourens et al., 2004;) e
Holoceno, de interesse para este trabalho, é caracterizado por mudanças climáticas cíclicas, marcadas por avanços e recuos do gelo sobre amplas áreas continentais e pelas variações eustáticas do nível do mar (Vicalvi, 1999). A maioria 
dos conhecimentos sobre mudanças paleoclimáticas ocorridas no Quaternário vem do estudo de seções sedimentares marinhas que, por se tratarem de ambientes cuja sedimentação se deu de maneira lenta e constante, fornecem um registro ideal do material orgânico utilizado como parâmetro nas reconstituições paleoambientais.

Estudos sobre o Quaternário das regiões costeiras brasileiras são comuns, mas o talude continental brasileiro ainda é pouco estudado. Estudos palinofaciológicos e organogeoquímicos de sequências sedimentares do talude continental da costa brasileira podem fornecer importantes informações sobre o registro paleoambiental e estão concentrados na área do talude continental recente da bacia de Campos, um local ideal para tais estudos, devido à ocorrência de taxas de sedimentação maiores que em outras áreas oceânicas (e.g. Menezes, 2002; Menezes \& Mendonça Filho, 2004).

Neste estudo foram utilizadas técnicas de microscopia em luz branca transmitida e luz azul incidente (fluorescência) para análise de palinofácies e métodos geoquímicos para análise do carbono orgânico total (COT), constituindo assim informações sobre os diferentes grupos dos componentes orgânicos particulados.

A partir da contagem do material orgânico particulado na análise palinofaciológica foram detectadas variações paleoambientais e paleoceanográficas, interpretadas com base nos diferentes conceitos que envolvem distribuição, sedimentação e preservação da matéria orgânica. Estas variações podem ser reflexos de oscilações do nível do mar e mudanças climáticas, influenciando na produção, sedimentação e preservação da matéria orgânica, afetando, assim, o suprimento de partículas orgânicas para o talude continental recente da bacia de Campos.

\section{MATERIAL E MÉTODOS}

A área de estudo compreende a porção norte do talude continental da bacia de Campos, localizada na parte sul do Estado do Espírito Santo, cerca de $75 \mathrm{~km}$ da linha de costa. O testemunho de sondagem BU91-GL-77 foi amostrado na profundidade aproximada de lâmina de água de $1.200 \mathrm{~m}$ (Figura 1). $O$ fundo do mar na região estudada tem relevo entrecortado por canais e apresenta declive suave $\left(0-3^{\circ}\right)$ (Kowsmann \& Vicalvi, 2003; Tokutake, 2005).

O testemunho de sondagem BU91-GL-77 (Figura 1), cedido pela PETROBRAS, constitui uma seção sedimentar quaternária do talude continental inferior da bacia de Campos, com 18,15 m de comprimento, seccionados em 85 amostras. Deste testemunho, foram confeccionadas 85 lâminas organopalinológicas e realizadas análises de COT em 75 destas amostras. A contagem de partículas orgânicas foi realizada em 68 lâminas, devido à ausência de material orgânico no restante das amostras.

A descrição das fácies sedimentares do testemunho, efetuada na escala 1:20, incluiu os seguintes parâmetros: cor, granulometria e estruturas primárias (Figura 2). A classificação de fácies combinou a textura do sedimento com o teor de $\mathrm{CaCO}_{3}$, este último estimado a partir da intensidade de reação em $\mathrm{HCl}$ 10\% (Kowsmann \& Vicalvi, 2003). Os teores de carbonatos foram os seguintes: margas (MG), com teores entre
30 e $60 \%$; lama rica em carbonato (LR), entre 30 e $15 \%$; lama levemente carbonática (LL), entre 15 e $5 \%$; e lama siliciclástica (L), entre 5 e $0 \%$.

A caracterização de fácies orgânica, empregada nesse estudo, aplicou a integração dos dados de palinofácies e geoquímica orgânica, seguindo o conceito de fácies orgânica de Tyson (1995).

A preparação das amostras para a análise de palinofácies seguiu os procedimentos não oxidativos para isolamento da matéria orgânica descritos por Tyson (1995), Mendonça Filho (1999) e Mendonça Filho et al. (2002). As amostras foram acidificadas com ácido clorídrico e fluorídrico e a matéria orgânica foi separada do resíduo mineral por diferença de densidade em relação ao cloreto de zinco. A matéria orgânica isolada foi então montada em lâminas de vidro para as análises em luz branca transmitida e fluorescência.

A análise de palinofácies envolveu o exame qualitativo e quantitativo da matéria orgânica, sendo o material orgânico dividido em três grupos principais: Matéria Orgânica Amorfa (MOA), Fitoclastos e Palinomorfos.

Dois sistemas de contagem foram realizados. O primeiro contabilizou o total de matéria orgânica (500 partículas), incluindo os três grupos - MOA, Fitoclastos e Palinomorfos, e o segundo contabilizou a origem marinha e continental da fração palinomorfo (300 partículas). As partículas orgânicas foram designadas a partir do sistema de classificação propostas por Tyson (1995), Mendonça Filho (1999) e Mendonça Filho et al. (2002).

\section{RESULTADOS}

Os valores percentuais dos constituintes da matéria orgânica (Fitoclasto, MOA e Palinomorfos) foram apresentados sob a forma de diagramas ternários (Figuras 3, 4) e sob a forma de gráfico com curvas de variação dos percentuais (Figura 5). De acordo com Tyson (1993, 1995), diagramas ternários são formas simples de representação gráfica, porém de um significado profícuo de apresentação de dados de palinofácies que podem mostrar um significante potencial para a discriminação de diferenças temporal e espacial em ambientes deposicionais.

A seção sedimentar estudada apresentou um predomínio dos grupos Fitoclastos (48,7\%) e Palinomorfos $(43,5 \%)$ no total dos componentes da matéria orgânica, sendo que MOA foi encontrado em pequeno percentual $(7,8 \%)$. O COT apresentou concentrações inferiores a $1 \%$ (mínimo de $0,25 \%$ ) ao longo de quase todo o testemunho, sendo que concentrações mais elevadas (máximo de 7,65\%) foram observadas apenas na base do testemunho.

Dentre os palinoformos, os de origem marinha foram dominantes, cujos percentuais atingiram valores superiores a $80 \%$ nos intervalos $1.500-800 \mathrm{~cm}$ e $600-0 \mathrm{~cm}$. Os palinomorfos continentais não ultrapassaram percentual de $40 \%$ e ao longo de todo o testemunho, tendo apresentado um comportamento bastante homogêneo no intervalo de $800-200 \mathrm{~cm}$.

A variável de grande importância que controla a tendência de distribuição dos grupos e subgrupos de componentes da matéria orgânica é a proximalidade continental (tendência proximal-distal). Este conceito de proximalidade, de grande utilidade em palinofácies, está interrelacionado (i) com a proxi- 
midade do ponto de origem de sedimentos siliciclásticos flúvio-deltaicos e da matéria orgânica terrestre (Fitoclastos), (ii) com a magnitude do ponto de origem flúvio-deltaico, (iii) com a magnitude e natureza da produtividade primária terrestre na área fonte do sedimento, (iv) com a duração relativa total do processo de transporte entre a área fonte das partículas e seu sítio final de deposição, e (v) com o gradiente paleoambiental entre a área fonte e o sítio final de deposição (Mendonça Filho, 1999; Menezes, 2002).

Com base nos três principais grupos de matéria orgânica (MOA, Fitoclastos e Palinoformos), Tyson (1993) propôs um diagrama ternário para classificação paleoambiental de ambientes marinhos proximais e distais em nove combinações que descrevem nove ambientes distintos. De acordo com os percentuais dos três principais grupos de matéria orgânica encontrados neste estudo, pode-se caracterizar a sucessão paleoambiental dos sedimentos estudados como variando, principalmente, de plataforma altamente proximal (I) a plataforma distal (V) (Figura 4).

As oscilações no suprimento de matéria orgânica terrestre para o ambiente marinho durante o Quaternário são relacionadas a flutuações climáticas deste período, que refletiram em alterações do nível relativo do mar. Correlacionando os paleoambientes identificados através do diagrama ternário com a coluna estratigráfica do poço, foi possível definir nove intervalos baseados nas tendências proximais-distais e traçar a curva relativa de variação do nível do mar para sucessão sedimentar estudada (Figura 5).

Intervalo 1. A palinofácies caracterizada corresponde à matéria orgânica típica de um paleoambiente variando de platafor- ma altamente proximal a uma plataforma óxica heterolítica. Apresenta alto suprimento de fitoclasto associado à ausência de MOA, indicando um ambiente proximal e de alta energia. Na base do perfil são concentrados sedimentos arenosos, condizentes com a equivalência hidrodinâmica dos fitoclastos que estão associados a partículas sedimentares grossas. Os maiores percentuais de COT foram encontrados neste intervalo, reflexo da abundância de matéria orgânica de origem terrestre, mais refratária. O intervalo é caracterizado por um nível do mar inicialmente mais baixo.

Intervalo 2. A matéria orgânica neste intervalo apresenta alto suprimento de fitoclasto associado à ausência de MOA e aumento de palinomorfos de origem continental, indicando um ambiente proximal e de alta energia, característica de um paleoambiente de plataforma óxica heterolítica. Em ambientes óxicos, os baixos valores de COT, observados neste intervalo e ao longo de todo o restante do testemunho, tendem a ser dominados por fitoclastos, os quais controlam a abundância do conteúdo de carbono. Ao final deste intervalo, os valores de COT e de palinomorfos continentais decrescem, indicando elevação relativa do nível do mar.

Intervalo 3. A matéria orgânica neste intervalo apresenta alto suprimento de palinomorfos de origem marinha, associada a um decréscimo acentuado nos percentuais de fitoclastos e baixos percentuais de MOA, caracterizando um ambiente de plataforma óxica dominada por lama (plataforma distal). Tais condições indicam uma tendência de elevação do nível do mar, que atingiu o máximo no final deste intervalo.

Intervalo 4. O paleoambiente de deposição da matéria orgânica neste intervalo volta a ser o de uma plataforma óxica

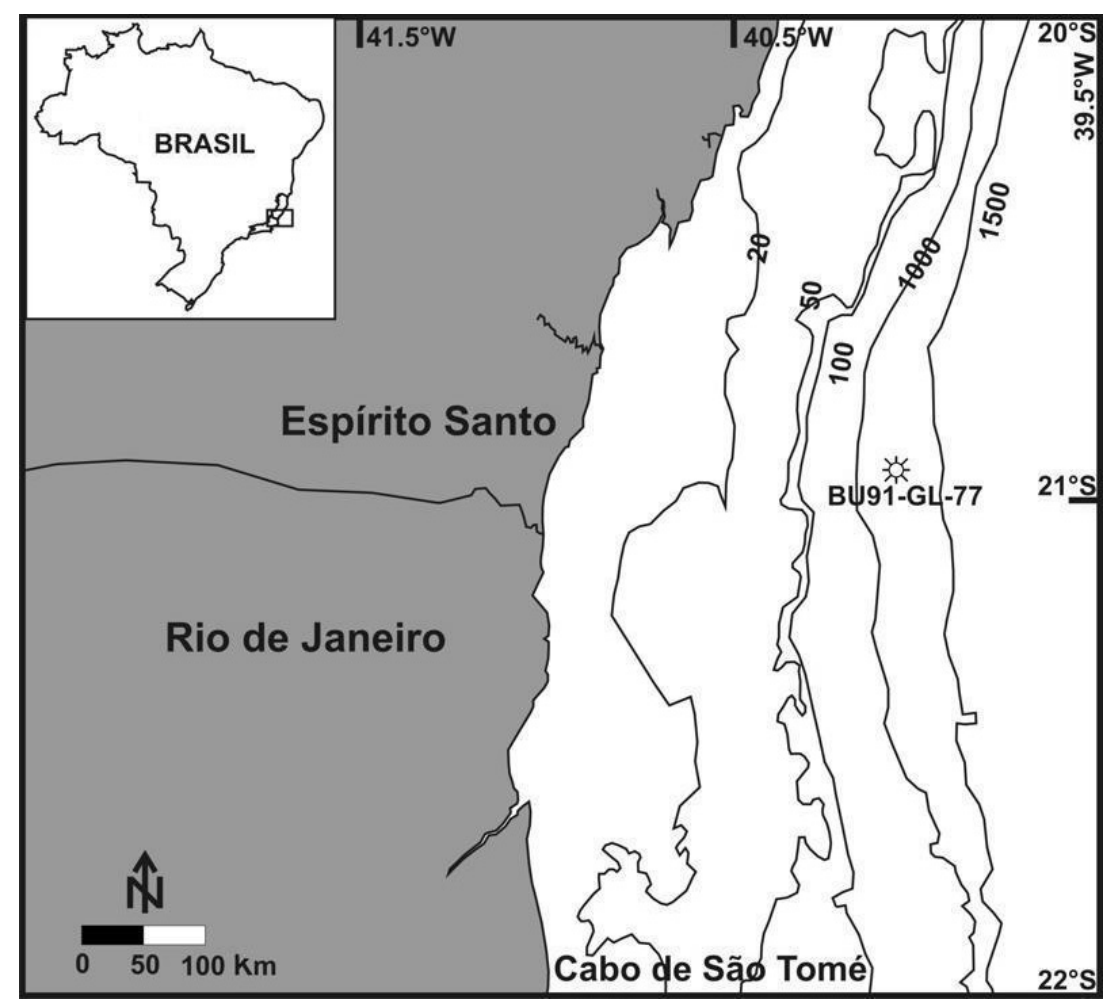

Figura 1. Mapa de localização do poço BU91-GL-77, bacia de Campos (modificado de Tokutake, 2005).

Figure 1. Location map of the well BU91-GL-77, Campos Basin (modified from Tokutake, 2005). 


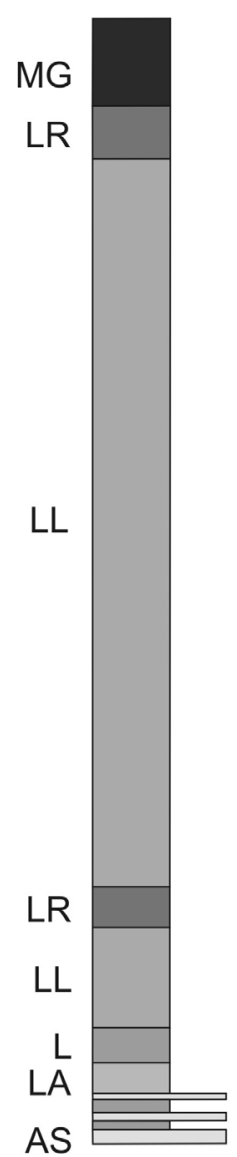

Legenda / Legend

(MG) marga amarelada a cinza oliva, bastante bioturbada

(Planolites, Chondrites, Thalassinóides e Zoophycos) /

Sufficiently bioturbated yellow to olive grey marl.

(LR) lama rica em carbornato, cinza oliva, bioturbada

(Planolites, Chondrites), com níveis oxidado /

Bioturbated, olive grey, carbonate rich mud with oxidised levels.

(LL) lama levemente carbonática, cinza esverdeada escuracom partes

mais claras, bioturbada (Chondrites, Zoophicos e Planolites / Bioturbated,

slightly carbonatic greenish dark grey mud with lighter color portions.

(L) lama siliciclástica, cinza esverdeada escura, bioturbada,micácea,

com níveis centimétricos ricos em matéria orgânica, e com tubos de pirita /

Micaceous, bioturbated greenish dark grey siliciclastic mud with centimetric levels rich in organic matter and with pyrite tubes.

(LA) lama arenosa, cinza esverdeada escura,micácea e bioturbada /

Bioturbated and micaceous greenish dark grey sandy mud.

(AS) areia siliciclástica composta de grãos de quartzo e mica, fino

a médio; base abrupta e topo bioturbado / Siliciclastic sand composed by fine

to medium quartz grains and mica; bottom is abrupt and top is bioturbated.

Figura 2. Descrição litofaciológica dos sedimentos recuperados no poço BU91-GL-77. Cores escolhidas arbritariamente.

Figure 2. Lithofaciologic description of sediments recovered from well BU91-GL-77. All colors have been chosen arbitrarily.

heterolítica (plataforma proximal). Há uma diminuição nos valores de palinomorfos de origem marinha e um acréscimo acentuado dos valores de fitoclastos, mostrando-se inverso aos valores do intervalo anterior. Tal situação indica uma tendência relativa de rebaixamento do nível do mar, tornando o ambiente mais proximal.

Intervalo 5. Neste intervalo, observa-se grande aumento nos percentuais de palinomorfos marinhos e decréscimo no percentual de fitoclasto, indicando novamente tendência de aumento relativo do nível do mar. A matéria orgânica novamente recai sobre um paleoambiente de plataforma óxica dominada por lama (plataforma distal) passando, no final deste intervalo, para uma plataforma distal disóxica-óxica. No final deste intervalo nota-se um aumento significante de MOA, indicativo de melhor preservação da matéria orgânica devido às condições redutoras e de baixa energia e sedimentação afastada da fonte de componentes terrestres, tendência essa que irá caracterizar o próximo intervalo.

Intervalo 6. Este intervalo é caracterizado pela queda nos percentuais de palinomorfos e elevada concentração de MOA, indicando condições redutoras na bacia. A análise de palinofácies indicou um paleoambiente cuja matéria orgânica foi depositada em uma plataforma proximal subóxica-anóxica, caracterizando tendência de rebaixamento do nível relativo do mar. Este pico de MOA pode estar relacionado a condições menos óxicas na bacia, causadas por possíveis áreas de confinamento pelo rebaixamento do nível do mar.
Intervalo 7. Deposição de matéria orgânica em um paleoambiente variando de uma bacia marginal disóxicaanóxica a uma plataforma distal disóxica-anóxica caracteriza este intervalo 7. O intervalo começa num paleoambiente onde, apesar da MOA tender a ser diluída principalmente pelo aumento no suprimento de fitoclasto, apresenta uma moderada taxa de preservação passando para um paleoambiente onde MOA também é dominante e apresentando baixos conteúdos de palinomorfos. A transição entre um ambiente e outro é marcada por um pequeno pico de fitoclasto, indicando alterações hidrodinâmicas decorrentes da elevação do nível do mar, tendência geral observada nesse intervalo.

Intervalo 8. A matéria orgânica volta a ser depositada em um paleoambiente caracterizado por uma plataforma óxica heterolítica (plataforma proximal). Há uma queda acentuada de MOA e o grupo fitoclasto aumenta junto com o grupo palinomorfo, sendo este último predominantemente de origem marinha. O paleoambiente deixa de ser um ambiente com características redutoras e passa a um ambiente cuja oxidação é comum. Ocorre uma pequena tendência de rebaixamento do nível do mar devido a essas novas condições.

Intervalo 9. A palinofácies caracterizada corresponde a uma matéria orgânica de um paleoambiente de plataforma óxica heterolítica (plataforma proximal) a uma plataforma óxica dominada por lama (plataforma distal). Apresenta redução do grupo de fitoclastos indicando distância das fontes flúviodeltaicas e abundantes palinomorfos de origem marinha, 


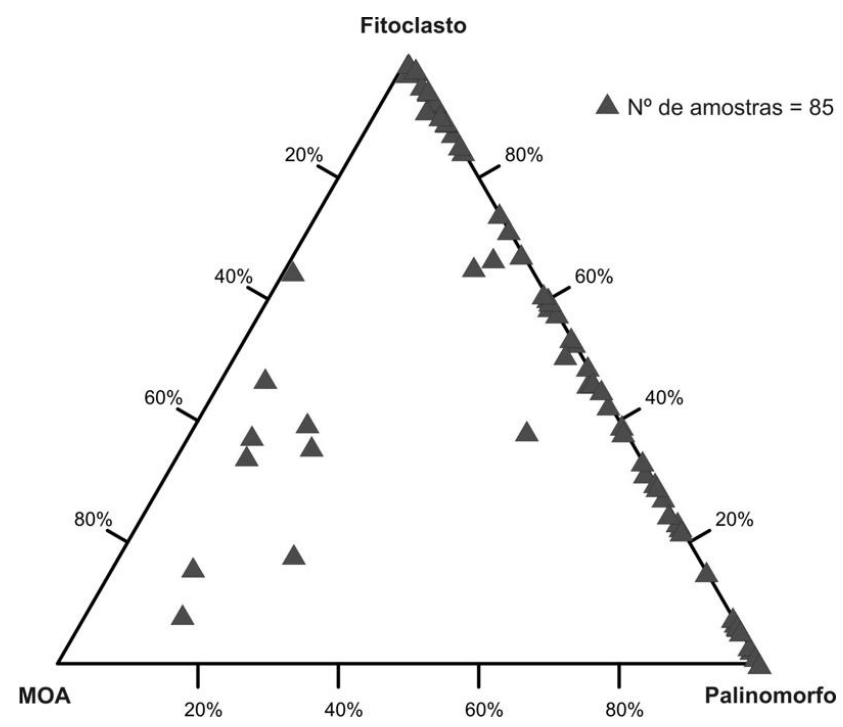

Figura 3. Diagrama ternário mostrando os percentuais de MOA, fitoclasto e palinomorfos das amostras analisadas do testemunho BU91-GL-77.

Figure 3. Ternary AOM-Phytoclast-Palynomorpf diagram of the analysed samples from core BU91-GL-77.

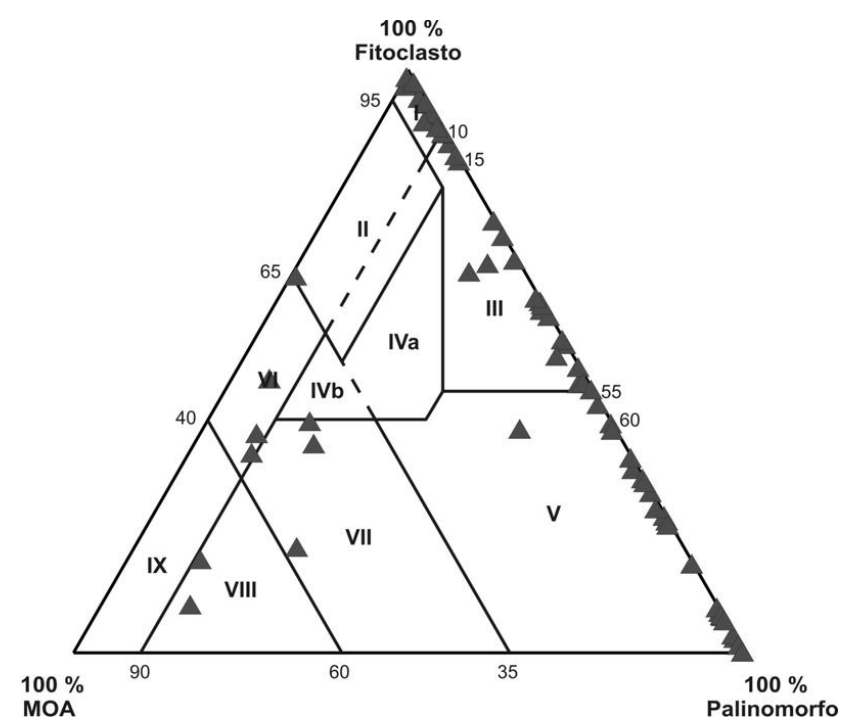

Figura 4. Diagrama ternário MOA-Fitoclasto-Palinomorfo (Tyson, 1995; Menezes et al., 2008) com os paleoambientes identificados com as amostras analisadas do testemunho BU91-GL-77. Abreviações: I, bacia ou plataforma altamente proximal; II, bacia marginal disóxica-anóxica; III, plataforma óxica heterolítica (plataforma "proximal"); IV, transição plataforma-bacia: IVa, disóxica, IVb, subóxica-anóxica; V, plataforma óxica dominada por lama (plataforma "distal"); VI, plataforma proximal subóxica-anóxica; VII, plataforma distal disóxica-anóxica; VIII, plataforma distal disóxicaóxica; IX, bacia distal subóxica anóxica/marinho restrito.

Figure 4. Ternary AOM-Phytoclast-Palinomorph organic matter plot (based on Tyson, 1993; Menezes et al., 2008) with identified paleoenvironments of the analysed samples from core BU91-GL77. Abbreviations: I, highly proximal shelf or basin; II, marginal dysoxic-anoxic basin; III, heterolithic oxic shelf ("proximal shelf"); IV, shelf to basin transition: IVa, dysoxic, IVb, suboxic-anoxic; V, muddominated oxic shelf ("distal shelf"); VI, proximal suboxicanoxic shelf; VII, distal suboxic-anoxic basin; VIII, distal dysoxicoxic shelf; IX, distal suboxic-anoxic basin/restricted marine. correspondentes aos seus maiores percentuais no perfil. Margas de coloração claras bioturbadas são comuns, o que confere com o perfil analisado. A tendência final é de elevação do nível relativo do mar para tal situação.

A curva de variação do nível relativo do mar, baseada na análise de palinofácies da sucessão sedimentar estudada, corrobora o proposto por Menezes \& Mendonça Filho (2004) e Vicalvi (1999). Estes autores analisaram, respectivamente, as paliniofácies e fácies orgânicas e a distribuição das espécies de foraminíferos planctônicos em um testemunho de sondagem amostrado no talude continental da bacia de Campos (150.000 anos AP) e observaram tendência geral de elevação do nível do mar, característico da passagem do Pleistoceno/Holoceno. Além disso, Menezes \& Mendonça Filho (2004) descreveram que esta tendência transgressiva é associada ao decréscimo na média percentual de fitoclastos e aumento de palinomorfos de origem marinha, MOA, COT e índice de IPF (Índice de Preservação do Fitoclasto). Neste trabalho, tanto o decréscimo no percentual de fitoclastos quando o aumento de palinomorfos de origem marinha foram observados, justificando assim a semelhança entre as curvas propostas de variação do nível relativo do mar para o talude continental da bacia de Campos.

\section{CONCLUSÕES}

O estudo da matéria orgânica particulada, análise de palinofácies e das características organogeoquímicas (COT) dos sedimentos do talude continental recente da bacia de Campos, mostrou que as variações do nível relativo do mar e as mudanças climáticas influenciaram diretamente a produção, sedimentação e preservação da matéria orgânica neste ambiente deposicional. Essas condições climáticas regem as variações de aporte e origem da matéria orgânica e de condições oxirredutoras do ambiente marinho de deposição, alterando assim as taxas de produção, acumulação e preservação da matéria orgânica sedimentada.

A caracterização paleoambiental da sucessão estudada, realizada a partir da plotagem dos dados de abundância relativa dos três grupos principais de matéria orgânica, em diagrama ternário, permitiu a identificação de nove paleoambientes com palinofácies distintas.

A matéria orgânica analisada nos sedimentos recuperados do poço BU91-GL-77 da bacia de Campos indica, da base para o topo, uma tendência geral de transgressão marinha marcada por pequenos ciclos de rebaixamento/elevação do nível relativo do mar em virtude das variações do influxo sedimentar continental, reflexos estes das flutuações climáticas do Quaternário. Os ciclos transgressivos no talude da bacia de Campos foram evidenciados pela caracterização de paleoambientes de plataforma óxica distal dominada por lama e plataforma distal disóxica-anóxica, enquanto que os ciclos regressivos ou de rebaixamento do nível do mar pelos paleoambientes de plataforma óxica heterolítica proximal e de bacia marginal disóxica-anóxica.

Os resultados obtidos com as técnicas de palinofácies e geoquímica orgânica usadas neste trabalho mostram que estes estudos constituem uma valiosa ferramenta no que diz respeito à interpretação paleoambiental e das condições de deposição da matéria orgânica sedimentar. 


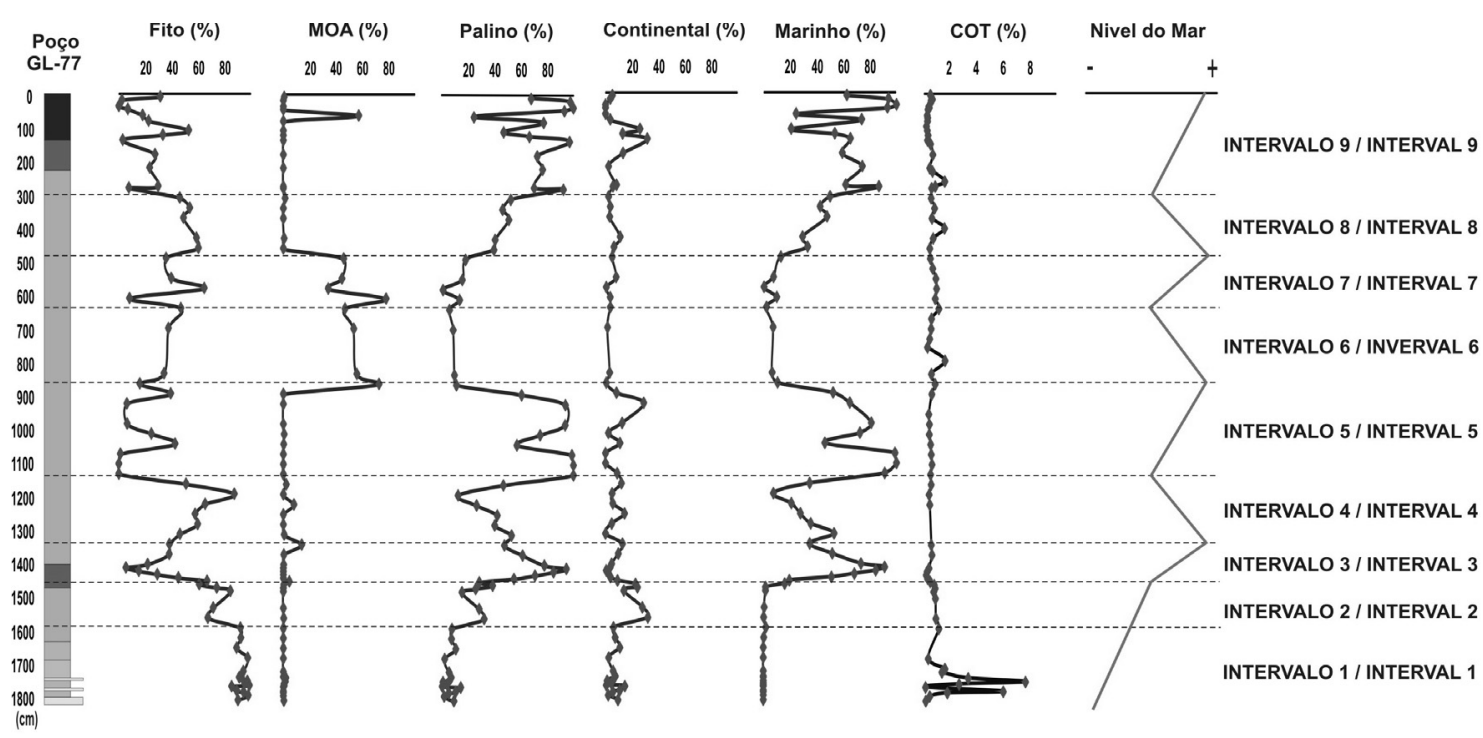

Legenda / Legend

(MG) marga amarelada a cinza oliva, bastante bioturbada (Planolites, Chondrites, Thalassinóides e Zoophycos) Sufficiently bioturbated yellow to olive grey marl.

(LR) Iama

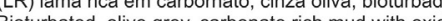

Bioturbated, olive grey, carbonate rich mud with oxidised levels.

(LL) lama levemente carbonática, cinza esverdeada escuracom partes mais claras, bioturbada (Chondrites, Zoophicos

e Planolites / Bioturbated, slightly carbonatic greenish dark grey mud with lighter color portions.

(L) lama siliciclástica, cinza esverdeada escura, bioturbada,micácea, com níveis centimétricos ricos em matéria orgânica, e com tubos de pirita / Micaceous, bioturbated greenish dark grey siliciclastic mud with centimetric levels rich in organic matter and with pyrite tubes.

LA) lama arenosa, cinza esverdeada escura, micácea e bioturbada

Bioturbated and micaceous greenish dark grey sandy mud.

(AS) areia siliciclástica composta de grãos de quartzo e mica, fino a médio; base abrupta e topo bioturbado / Siliciclastic sand

composed by fine to medium quartz grains and mica; bottom is abrupt and and top is bioturbated.

Figura 5. Variação dos parâmetros palinofaciológicos e organogeoquímicos utilizados para traçar a curva do nível relativo do mar do testemunho BU91-GL-77.

Figure 5. Palynofacies and organic geochemical parameters showing the sea level change of core BU91-GL-77.

\section{AGRADECIMENTOS}

À equipe do Laboratório de Palinofácies e Fácies Orgânica-LAFO, Departamento de Geologia, Instituto de Geociências, UFRJ.

\section{REFERÊNCIAS}

Gibbard, P. \& Van Kolfschoten, T. 2004. The Pleistocene and Holocene epochs. In: F.M. Gradstein; J.G. Ogg \& A.G. Smith (eds.) A Geologic Time Scale 2004, Cambridge University Press, p. 441-452.

Kowsmann, R.O. \& Vicalvi, M.A. 2003. Descrição e datação dos furos da campanha Bucentaur 2003 na área de Jubartel Cachalote (Bloco BC-60). PDEXP/PETROBRAS, Relatório Interno RT GEOF n ${ }^{\circ} 008 / 2003,17$ p.

Lourens, L.; Hilgen, F.; Shackeleton, N.J.; Laskar, J. \& Wilson, D. 2004. The Neogene period. In: F.M. Gradstein; J.G. Ogg \& A.G. Smith (eds.) A Geologic Time Scale 2004, Cambridge University Press, p. 409-440.

Mendonça Filho, J.G. 1999. Aplicação de estudos de palinofácies e fácies orgânica em rochas do Paleozóico da bacia do Paraná, sul do Brasil. Programa de Pós-Graduação em Geociências, Universidade Federal do Rio Grande do Sul, Tese de Doutorado, $338 \mathrm{p}$.

Mendonça Filho, J.G; Carvalho, M.A. \& Menezes, T.R., 2002. Palinofácies. In: T.L. Dutra (org.) Técnicas e procedimentos de trabalho com fósseis e formas modernas comparativas, Editora Unisinos, p. 20-24.
Menezes, T.R., 2002. Aplicação de parâmetros palinofaciológicos e organogeoquímicos na reconstrução paleoambiental do talude continental brasileiro Recente na Bacia de Campos - RJ. Programa de Pós-Graduação em Geologia, Universidade Federal do Rio de Janeiro, Dissertação de Mestrado, 174 p.

Menezes, T.R. \& Mendonça Filho, J.G. 2004. Aplicação de fácies orgânica na análise paleoceanográfica do talude continental superior Recente da Bacia de Campos, RJ. Revista Brasileira de Paleontologia, 7(2):177-188.

Menezes, T.R.; Mendonça Filho, J.G.; Araujo, C.V.; Souza, I.V.A.F. \& Mendonça, J.O. 2008. Fácies orgânica: conceitos, métodos e estudos de casos na indústria do petróleo. Revista Brasileira de Geociências, 38(2-supl.):1-17.

Tokutake, L.R. 2005. Bioestratigrafia de nanofósseis calcários e estratigrafia de isótopos ( $\mathrm{Ce} \mathrm{O})$ do talude médio, Quaternário, porção N da bacia de Campos, ES. Programa de Pós-graduação em Geociências, Universidade Federal do Rio Grande do Sul, Dissertação de Mestrado, 97 p.

Tyson, R.V. 1993. Palynofacies analysis. In: D.J. Jenkins (ed.) Applied micropaleontology, Kluwer Academic Publishers, p. 153-191.

Tyson, R.V. 1995. Sedimentary organic Matter: Organic facies and palynofacies. $1^{\mathrm{a}}$ ed. Londres, Chapman \& Hall, $615 \mathrm{p}$.

Vicalvi, M.A.1999. Zoneamento bioestratigráfico e paleoclimático do Quaternário Superior do talude da bacia de Campos e platô de São Paulo adjacente, com base em foraminíferos planctônicos. Programa de Pós-Graduação em Geologia, Universidade Federal do Rio de Janeiro, Tese de Doutorado, 184 p.

Received in June, 2009; accepted in March, 2010. 